Western University

Scholarship@Western

Electrical and Computer Engineering

Electrical and Computer Engineering

Publications

Department

Winter 2-1-2020

\title{
A lightweight magnetorheological actuator using hybrid magnetization
}

Masoud Moghani

The University of Western Ontario

Mehrdad Kermani Ph.D., P.Eng.

Western University, mkermani@eng.uwo.ca

Follow this and additional works at: https://ir.lib.uwo.ca/electricalpub

Part of the Computer Engineering Commons, and the Electrical and Computer Engineering Commons

Citation of this paper:

M. Moghani and M. R. Kermani, "A Lightweight Magnetorheological Actuator Using Hybrid Magnetization," in IEEE/ASME Transactions on Mechatronics, vol. 25, no. 1, pp. 76-83, Feb. 2020, doi: 10.1109/

TMECH.2019.2951340. 


\title{
A Lightweight Magneto-Rheological Actuator using Hybrid Magnetization
}

\author{
Masoud Moghani, and Mehrdad R. Kermani, Member, IEEE
}

\begin{abstract}
This paper presents the design and validation of a lightweight Magneto-Rheological (MR) clutch, called Hybrid Magneto-Rheological (HMR) clutch. The clutch utilizes a hybrid magnetization using an electromagnetic coil and a permanent magnet. The electromagnetic coil can adjust the magnetic field generated by the permanent magnet to a desired value, and fully control the transmitted torque. To achieve the maximum torque to mass ratio, the design of HMR clutch is formulated as a multiobjective optimization problem with three design objectives, namely the transmitted torque, the mass of the clutch, and the magnetic field strength within the clutch pack. A prototype of the HMR clutch is fabricated and its dynamic performance is experimentally validated. Experimental results clearly demonstrate the advantages of the HMR clutch in applications requiring fast and precise motion and torque control.
\end{abstract}

Index Terms-Magnetorheological actuator, hybrid magnetization.

\section{INTRODUCTION}

$\mathbf{M}$ AGNETO-RHEOLOGICAL (MR) fluids are functional fluids whose viscosity can change rapidly, continuously, and reversibly in response to an applied magnetic field. The ability to change the viscosity of MR fluids rapidly and precisely gives MR devices desirable characteristics for control and robotic applications.

A wide range of studies have looked at the design of MR devices. A comparison of different MR clutch configurations for their compactness, power consumption, and torque transmission were presented in [1] and [2]. An analytical method for design optimization of MR brakes with a desired magnetic flux over the MR fluid surface was developed in [3]. A multiobjective optimization technique was employed in [4], aiming at maximizing the braking torque to weight ratio. An advanced objective function consisting of the damping force, dynamic range, and inductive time constant of an MR damper was considered in [5]. The work extended the results of a previous study [6], in which only the power consumption of the MR damper was considered as an objective function. Other studies focused on the development of high torque MR clutches [7][10]. The design of a conventional MR clutch was reported in [11] for a clutch with $75 \mathrm{Nm}$ torque, weighing $4.5 \mathrm{~kg}$. Kikuchi et al. [12] achieved impressive results for a compact MR clutch that produced $40 \mathrm{Nm}$ of torque and weighed 2.2 $\mathrm{kg}$. In this design, the gaps between the disks were reduced

M. Moghani was with the Department of Electrical and Computer Engineering, the University of Western Ontario, London, ON N6A 5B9, Canada (e-mail: mmoghan@uwo.ca)

M. R. Kermani is with the Department of Electrical and Computer Engineering, the University of Western Ontario, London, ON N6A 5B9, Canada (e-mail: mkermani@eng.uwo.ca) significantly comparing to regular disk-based MR clutches to decrease the reluctance of the magnetic path. Yadmellat et al. [13] used antagonistic MR clutches in a safe 2-DOF manipulator. The MR clutches were able to achieve $15 \mathrm{Nm}$ of torque and weighed $2.3 \mathrm{~kg}$. The dual differential rheological actuator (DDRA) was in [14]. This actuator coupled two different MR brakes, each of which moved at the same speed but in opposite directions. The coupled MR brakes generated $20 \mathrm{Nm}$ of maximum torque and weighed $2.4 \mathrm{~kg}$.

In most MR devices, electromagnetic coils are used as the primary source of magnetic field generation and the use of permanent magnets alone or in combination with electromagnetic coils has received less attention. A fail-safe MR device excited by permanent magnets was presented in [15]. The device was designed to engage and disengage a power brake vacuum pump from a diesel engine by positioning a permanent magnet inside the device. An MR valve using a combination of a permanent magnet and an electromagnetic coil was proposed in [16]. A normally closed (fully engaged) MR clutch using permanent magnets and an electromagnetic coil for use in human-robot interactions was studied in [17]. Under normal conditions, the clutch transmitted maximum torque under the influence of the permanent magnets. To disengage the clutch, the electromagnetic coil was energized to nullify the magnetic field of the permanent magnets. MR clutches for high rotational velocities using different combinations of permanent magnets and electromagnetic coils were presented in [18]. Three different MR clutches were studied for medium and high torque transmission.

This paper presents design, optimization and validation of an MR clutch with hybrid magnetization, called Hybrid Magneto-Rheological (HMR) clutch. A combination of a permanent magnet ring and an electromagnetic coil provides the magnetic field inside the clutch. This configuration allows distributing the magnetic flux inside the clutch uniformly, thereby reducing magnetic saturation in the magnetic path. By avoiding saturation, the volume of the material in the magnetic path as well as the total mass and inertia of the clutch are reduced. The new design improves the torque to mass ratio of the HMR clutch making this device a suitable candidate for designing high-performance actuators.

The organization of this paper is as follow: Section II introduces the design of the HMR clutch and discusses parameter selections of the clutch. Section III presents dynamic characteristics and experimental validations of a prototype HMR clutch. Finally, Section IV concludes the paper. 


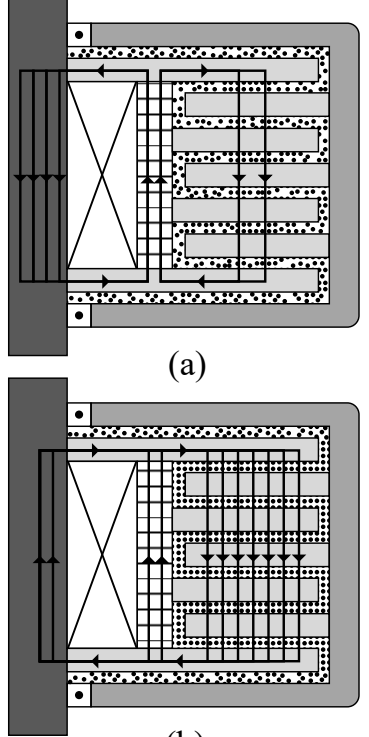

(b)
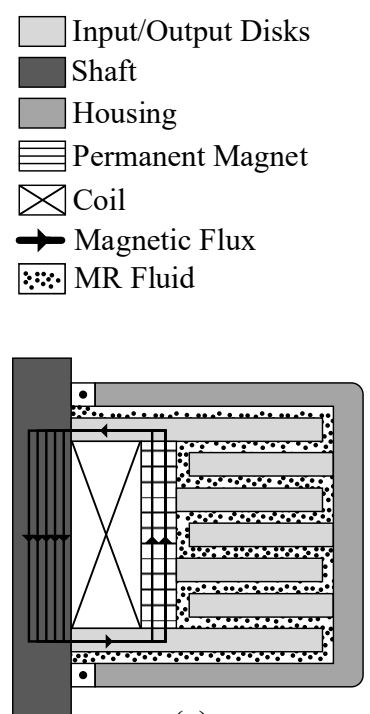

(c)
Fig. 1. Magnetic field distribution inside the HMR clutch: (a) magnetic flux distribution of a permanent magnet, (b) full magnetization with permanent magnet and electromagnetic coil, (c) magnetic field cancellation within the clutch pack with induced magnetic field in reverse direction.

\section{Hybrid Magneto-Rheological Clutch}

Unlike conventional MR clutches that use an electromagnetic coil to generate the magnetic field, the HMR clutch uses a combination of a permanent magnet and an electromagnetic coil for generating the magnetic field. We use this approach with a careful selection of a permanent magnet and an electromagnetic coil size to provide an optimum design solution for the HMR clutch. To this end, we use a permanent magnet to partially engage the HMR clutch (Fig. 1(a)). In other words, the permanent magnet provides the clutch with an initial magnetic field. The electromagnetic coil is used to adjust the strength of the magnetic field within the clutch pack to a desired value on demand. Depending on the polarity of the input current, the magnetic field of the electromagnetic coil can be either added to or subtracted from that of the permanent magnet (Fig. 1(b) and (c)). This is a novel approach allowing to generate maximum torque and absolute zero torque in HMR clutches while achieving the highest torque to mass ratio.

One significant advantage of this approach is to enable higher input currents and larger torques without magnetic saturation thanks to uniform distribution of the magnetic flux. Put differently, for a given current input, an HMR clutch weighs substantially due to less volume of ferromagnetic material in the magnetic circuit.

\section{A. Figures of Merit}

In this section, an analytical model of the HMR clutch is developed using the parameters shown in Fig. 2(a). It is assumed that the clutch has $N$ output disks coupled to the output part, $N-1$ input disks coupled to the input shaft. $R_{1} / R_{2}$ are inner and outer radii of the input/output disks, and $t_{d}$ is the thickness of the disks. $R_{s h}$ is the radius of the main

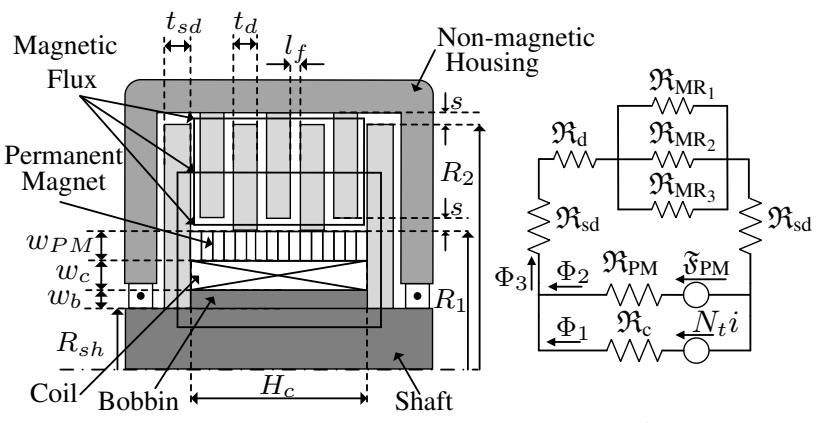

(a)

(b)

Fig. 2. (a) Geometry and (b) equivalent magnetic circuit of a typical HMR clutch

shaft. $w_{b}$ is the width of the bobbin, $w_{c}$ is the width of the electromagnetic coil, and $w_{P M}$ is the width of the permanent magnet. $H_{c}$ is the length of the clutch pack, $t_{s d}$ is the thickness of the side disks, and $l_{f}$ is the thickness of the MR fluid gap. In total, the clutch pack contains $2 N-1$ disks and $2 N$ MR fluid gaps.

1) Magnetic Circuit: Here, the reluctance of different parts of the magnetic circuit shown in Fig. 2(b) are obtained. In this model, $\mathfrak{R}_{\mathrm{c}}, \mathfrak{R}_{\mathrm{sd}}, \mathfrak{R}_{\mathrm{d}}$, and $\mathfrak{R}_{\mathrm{MR}}$ are the reluctance of the core, side disk, inner/outer disks, and MR fluid gap, respectively. The reluctance of a uniform magnetic circuit is given as $\mathfrak{R}=l /\left(\mu_{0} \mu_{r} A\right)$, where $l$ is the mean length of the magnetic circuit, $\mu_{0}$ is the permeability of free space, $\mu_{r}$ is the relative permeability of the material, and $A$ is the cross section area of the material. The magnetic reluctance of each part of the HMR clutch can be derived as,

$$
\begin{aligned}
\mathfrak{R}_{\mathrm{c}} & =\frac{H_{c}+t_{s d}}{\mu_{0} \mu_{s} \pi\left(R_{s h}\right)^{2}} \quad \mathfrak{R}_{\mathrm{d}}=\frac{t_{d}}{\mu_{0} \mu_{s} \pi\left(\left(R_{2}\right)^{2}-\left(R_{1}+s\right)^{2}\right)} \\
\mathfrak{R}_{\mathrm{sd}} & =\int_{R_{s h}}^{\frac{1}{2}\left(R_{2}+R_{1}\right)} \frac{d r}{\mu_{0} \mu_{s}(2 \pi r) t_{s d}}=\frac{\ln \left(\frac{R_{2}+R_{1}}{2 R_{s h}}\right)}{2 \mu_{0} \mu_{s} \pi t_{s d}}
\end{aligned}
$$

where $\mu_{s}$ is the permeability of steel, used in the construction of the core, side disks, and inner/outer disks. The reluctance of the clutch pack $\mathfrak{R}_{\mathrm{cp}}$ is given as,

$$
\mathfrak{R}_{\mathrm{cp}}=(2 N-1) \mathfrak{R}_{\mathrm{d}}+\mathfrak{R}_{\mathrm{MR}}
$$

where $\mathfrak{R}_{\mathrm{MR}}$ is the reluctance of the MR fluid that includes three parts as shown in Fig. 2(b) and is formulated as [19],

$$
\left(\mathfrak{R}_{\mathrm{MR}}\right)^{-1}=\left(\mathfrak{R}_{\mathrm{MR}_{1}}\right)^{-1}+\left(\mathfrak{R}_{\mathrm{MR}_{2}}\right)^{-1}+\left(\mathfrak{R}_{\mathrm{MR}_{3}}\right)^{-1}
$$

in that $\mathfrak{R}_{\mathrm{MR}_{1}}=\frac{2 N l_{f}+(N-1) t_{d}}{\mu_{0} \mu_{m} \pi\left(\left(R_{2}+s\right)^{2}-\left(R_{2}\right)^{2}\right)}, \mathfrak{R}_{\mathrm{MR}_{2}}=\frac{2 N l_{f}}{\mu_{0} \mu_{m} \pi\left(\left(R_{2}\right)^{2}-\left(R_{1}+s\right)^{2}\right)}$, and $\mathfrak{R}_{\mathrm{MR}_{3}}=\frac{2 N l_{f}+N t_{d}}{\mu_{0} \mu_{m} \pi\left(\left(R_{1}+s\right)^{2}-\left(R_{1}\right)^{2}\right)}$ represent the reluctance of each path of the MR fluid, and $\mu_{m}$ is the permeability of the MR fluid. The permanent magnet with linear demagnetization curve is modeled as a series combination of a reluctance $\mathfrak{R}_{\mathrm{PM}}$ with a magnetomotive force $\mathfrak{F}_{\text {PM }}$ defined as [1],

$$
\mathfrak{F}_{\mathrm{PM}}=\frac{\mathfrak{B}_{\mathrm{r}} l_{P M}}{\mu_{0}} \quad \text { and } \quad \mathfrak{R}_{\mathrm{PM}}=\frac{l_{P M}}{\mu_{0} A_{P M}}
$$

where $l_{P M}=H_{c}$ is the length, $A_{P M}$ is the surface area, and $\mathfrak{B}_{\mathrm{r}}$ is the remanent flux density of the permanent magnet. 


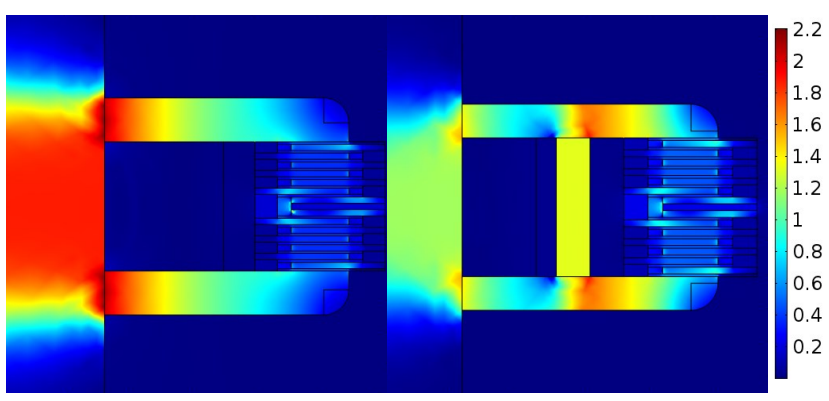

(a)

(b)

Fig. 3. Magnetic flux density in (a) conventional MR clutch, and (b) HMR clutch.

Using Kirchhoff's laws in Fig. 2(b) one can easily obtain the flux $\Phi_{3}$ within the clutch pack as,

$$
\Phi_{3}=\frac{\mathfrak{R}_{\mathrm{PM}}\left(N_{t} i\right)+\mathfrak{R}_{\mathrm{c}}\left(\mathfrak{F}_{\mathrm{PM}}\right)}{\mathfrak{R}_{\mathrm{PM}} \mathfrak{R}_{\mathrm{c}}+\left(\mathfrak{R}_{\mathrm{cp}}+2 \mathfrak{R}_{\mathrm{sd}}\right) \times\left(\mathfrak{R}_{\mathrm{PM}}+\mathfrak{R}_{\mathrm{c}}\right)}
$$

where $i$ is the total electric current, and $N_{t}$ is the number of turns in the coil. Finally, the magnetic flux density $\mathfrak{B}_{\mathrm{MR}}$ in the MR fluid path is computed as,

$\mathfrak{B}_{\mathrm{MR}}=\frac{\Phi_{3}}{\pi\left(\left(R_{2}\right)^{2}-\left(R_{1}+s\right)^{2}\right)} \frac{\mathfrak{R}_{\mathrm{MR}_{1}} \mathfrak{R}_{\mathrm{MR}_{3}}}{\mathfrak{R}_{\mathrm{MR}_{1}}\left(\mathfrak{R}_{\mathrm{MR}_{2}}+\mathfrak{R}_{\mathrm{MR}_{3}}\right)+\mathfrak{R}_{\mathrm{MR}_{2}} \mathfrak{R}_{\mathrm{MR}_{3}}}$

where only the central path along the clutch pack is considered in obtaining the magnetic flux density. This is due to the fact that the overlapped area of the inner/outer disks can contribute in torque transmission [19].

To analyze the behavior of the proposed HMR clutch, COMSOL Multiphysics® software was used on a finite element model of the system. Fig. 3 compares a conventional MR clutch (left) with the HMR clutch (right). The magnetic flux density contours in both types of the clutches are shown for an input current of $2 \mathrm{~A}$. As seen, the conventional MR clutch is highly saturated near the intersection of the shaft and the side disks. However, the HMR clutch shows a more uniform distribution of magnetic flux which alleviates saturation. Detailed comparison of the two types of clutches is reported in [20] and is not repeated here for brevity.

2) Torque Computation: The strength of the magnetic field determines the sheer stress developed by the MR fluid. The sheer stress $\tau=\tau_{y}(H)+\eta \frac{d v}{d z}$ is governed by Bingham viscoplastic model [21], where $\tau_{y}$ is the field-dependant yield stress, $H$ is the applied magnetic field, $\eta$ is the Newtonian viscosity, and $d v / d z$ is the velocity gradient in the direction of the field. The velocity gradient is often assumed constant under the conditions discussed in [11] and is expressed as $d v / d z=\omega r l_{f}^{-1}$, in that $\omega$ is the angular velocity between the input and output shafts, and $r$ is the radius from the rotational axis. Integrating the sheer stress over the entire disk area yields the transmitted torque of the HMR clutch as,

$$
T=4 N \pi\left(\frac{\tau_{y}(H)\left(R_{2}^{3}-R_{1}^{3}\right)}{3}+\frac{\eta \omega\left(R_{2}^{4}-R_{1}^{4}\right)}{4 l_{f}}\right)
$$

The field dependent yield stress used in (6) can be expressed in terms of the magnetic field intensity applied to the MR fluid and the material properties of the MR fluid [22] as,

$$
\tau_{y}(H)=2.717 \times 10^{5} C \phi^{1.5239} \tanh \left(6.33 \times 10^{-6} H\right)
$$

where $\phi$ is the volume fraction of iron and $C$ is a constant that depends on the carrier fluid (e.g., $C=1$ for hydrocarbon oil).

3) Actuator Mass: The lower mass of the proposed HMR clutch is an important consideration during the design procedure. Using the geometry of a typical HMR clutch shown earlier in Fig. 2(a), the total mass of the clutch can be obtained as,

$$
M=\rho_{s}\left(V_{s h}+V_{b}+V_{s d}+V_{d}\right)+\rho_{p m} V_{p m}+\rho_{c u} V_{c o i l}
$$

where $\rho_{s}, \rho_{p m}$, and $\rho_{c u}$ are the densities of steel, permanent magnet, and copper, respectively, and $V_{s h}, V_{b}, V_{s d}, V_{d}, V_{p m}$, and $V_{\text {coil }}$ are the volumes of the shaft, bobbin, side disks, inner/outer disks, permanent magnet, and electromagnetic coil, respectively.

\section{B. Design Optimization using Finite Element Analysis}

We use the values of the parameters of the HMR clutch obtained in the previous sections and the numerical values of the material properties in order to model and optimize the design of the HMR clutch. COMSOL Multiphysics® software is used for finite element (FE) modeling and optimization. A 2D model is built to provide information about the intensity of the magnetic field inside the HMR clutch. The magnetic properties of different materials used in the construction of the clutch is included in the model in the form of B-H curves. The model includes the relationship between the input current, the magnetic field, and the output torque of the clutch.

The optimization problem is defined to maximize the output torque (i.e., $\tau_{d}$ ) of the clutch and minimize the total mass (i.e., $m_{t}$ ), and magnetic field nullification within the clutch pack (i.e., $f_{n u l}$ ). These variables are used to define the following objective function for obtaining the optimal design parameters of the HMR clutch, i.e.,

$$
\min \left(\left|\tau_{d}-\tau_{s}\right|, m_{t},\left|f_{n u l}\right|\right)
$$

where $\tau_{s}$ represents the nominal output torque. The optimization problem is subject to a set of constraints that represent the physical limits of the clutch parameters as followings,

- maximum current density of the coil given its temperature limits.

- physical relations between the dimensions (e.g., $R_{1}=$ $\left.R_{s h}+w_{b}+w_{c}+w_{P M}, R_{2}>R_{1}\right)$.

- availability of the material (e.g., standard steel sheets, permanent magnet grade, etc.)

- machining and manufacturing limitations (e.g., permanent magnet thickness, MR fluid gap, inner housing thickness).

- a fixed shaft radius of $15 \mathrm{~mm}$.

A full factorial design is used to obtain a unique configuration for every possible combination of the design parameters. Different ranges of the design parameters (e.g., side disk thickness, bobbin width, coil size, permanent magnet size, 


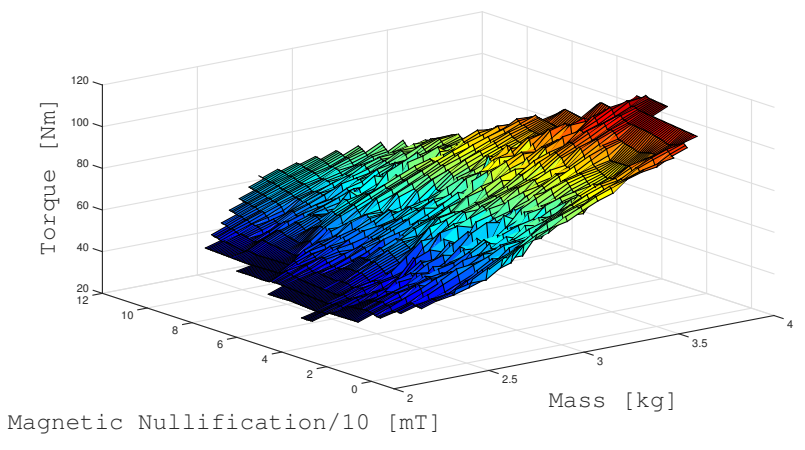

Fig. 4. Achievable output torque vs. mass and magnetic field nullification

disks overlapping area, etc.) are examined first to obtain the convergence ranges. The constrained ranges are then used in COMSOL Multiphysics® software. The solution of the optimization problem provides the optimal dimensions of the HMR clutch.

The radius of the shaft was set to $15 \mathrm{~mm}$ to be compatible with an existing setup used for a conventional MR clutch. However, it was possible to use a smaller shaft as shown in [20] since the shaft of the HMR clutch is less susceptible to magnetic saturation. To avoid the need for manufacturing parts with high tolerances, we increased overlap area between the input/output disks (e.g., $R_{2}-R_{1}$ ). This contributed to an increased transmission torque. Last, we limited the results to those that led to an absolute magnetic nullification. Fig. 4 shows the maximum achievable output torque versus the mass and magnetic field nullification. As seen, a maximum torque of $65 \mathrm{Nm}$ and a nullified magnetic field of $0 \mathrm{mT}$ correspond to a total mass of $2.6 \mathrm{~kg}$. This solution was selected for further development and prototyping.

The magnetic flux density of the HMR clutch designed in the previous section is shown in Fig. 5. The figure includes four different values of the input currents. As seen in Fig. 5(a), for an input current of $-1 \mathrm{~A}$, the electromagnetic coil nullifies the magnetic field of the permanent magnet within the clutch pack with zero output torque in the clutch (fully decoupled). An input current of $0 \mathrm{~A}$ shown in Fig. 5(b) results in the magnetic flux density of the permanent magnet to pass mostly through the shaft due to its small reluctance compared to the clutch pack. Fig. 5(c) and (d) show the magnetic flux densities for input currents of $2 \mathrm{~A}$ and $4 \mathrm{~A}$, respectively. These input currents result in $43 \mathrm{Nm}$ and $55 \mathrm{Nm}$ torques according to the model of the HMR clutch. We will show next that these values closely match the experimental data. These are absolute torque values generated by the HMR clutch with no use of any gear reduction.

\section{EXPERIMENTAL VALIDATION OF HMR ClutCH}

The final design of the HMR clutch is shown in Fig. 6. The input/output disks are separated using nonmagnetic spacers. The electromagnetic coil is wound on a magnetic bobbin that is keyed to the output shaft. An N45 Neodymium permanent magnet ring that is magnetized along its rotational axis is used

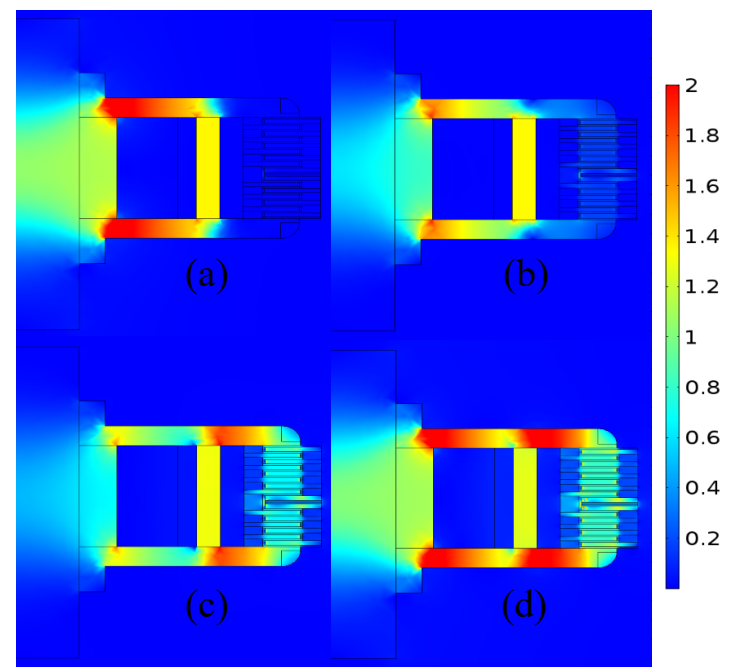

Fig. 5. Magnetic flux density in the HMR clutch for (a) $-1 \mathrm{~A}$, (b) $0 \mathrm{~A}$, (c) 2 A, and (d) 4 A excitation current.

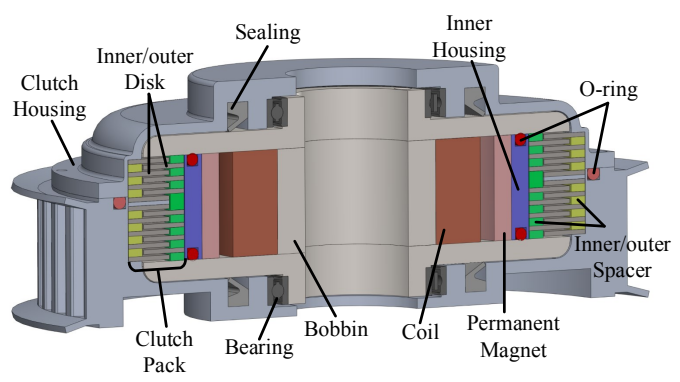

Fig. 6. Assembly of the HMR clutch.

inside the clutch. To protect the permanent magnet against corrosion, the magnet is coated. The specifications of the prototype HMR clutch are listed in Table I.

TABLE I

SPECIFICATIONS OF THE HMR CLUTCH

\begin{tabular}{lc}
\hline \hline Diameter [mm] & 155 \\
Width [mm] & 59 \\
MR fluid gap thickness [mm] & 0.425 \\
No. of input disks & 8 \\
Torque at 0 A [Nm] & 4.5 \\
Maximum torque [Nm] & 65 \\
Total mass with housing [kg] & 3.1 \\
Torque density [Nm/kg] & 21 \\
\hline \hline
\end{tabular}

To demonstrate the advantage of our proposed design, Fig. 7 compares the torque density (torque to mass ratio) of the HMR clutch presented in this work with those previously reported in the literature. The results clearly show the superiority of the proposed HMR clutch. As seen in Fig. 7, the torque density of our proposed HMR clutch using a combination of a permanent magnet and an electromagnetic coil is almost twice of that of a clutch with the same design and no permanent magnet. 


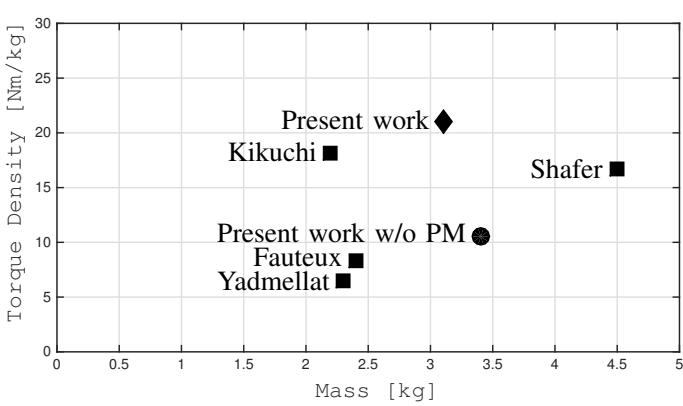

Fig. 7. Comparison of torque density and mass of the HMR and conventional MR clutches. Source: Shafer et al. [11], Kikuchi et al. [12], Fauteux et al. [14], Yadmellat et al. [13].

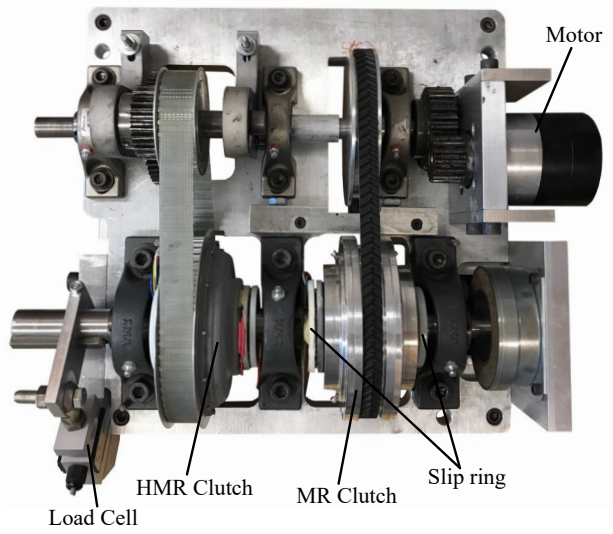

Fig. 8. Experimental test bench for testing the prototype HMR clutch

The experimental test bench used to evaluate the functionality of the prototype HMR clutch is shown in Fig. 8. In this setup, one HMR clutch and one conventional MR clutch were used in an antagonistic configuration. The test bench included a servo motor (Maxon EC 60) to provide the input torque to the clutches. A high-power motor driver (Maxon Motors ADS 50/5 servo amplifier) set in the current mode, provided the input current to the HMR clutch. A static load cell (Transducer Techniques SBO-1K) measured the output torque of the prototype HMR clutch. An angular encoder (Renishaw RM22I) was used to read the position of the output shaft. Two unipolar ratiometric analog Hall sensors (Infinion TLE4990) and two bipolar digital Hall sensors (Infinion TLE4998S) faced in opposite directions were embedded inside the clutch to measure magnetic flux density up to $\pm 400 \mathrm{mT}$. As shown in Fig. 5, the predicted magnetic flux density should remain within $\pm 400 \mathrm{mT}$ range of the embedded Hall sensors. A dSPACE (DS 1103, dSPACE Inc.) controller board was used to acquire the data and to command suitable control signals to the clutches.

To measure the torque capacity of the HMR clutch, a sinusoidal current with the frequency of $1 \mathrm{~Hz}$ and magnitude of $2 \mathrm{~A}$ and a DC component of $1.3 \mathrm{~A}$ varying between $-0.7 \mathrm{~A}$ and 3.3 A was applied to the prototype HMR clutch. The input side of the clutch was driven at a constant rotational velocity while its output side was locked to a stationary load cell. Fig. 9 depicts

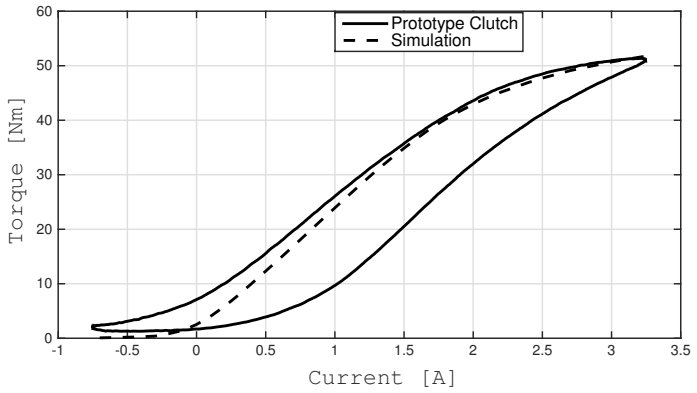

Fig. 9. Comparison between measured and simulated results.

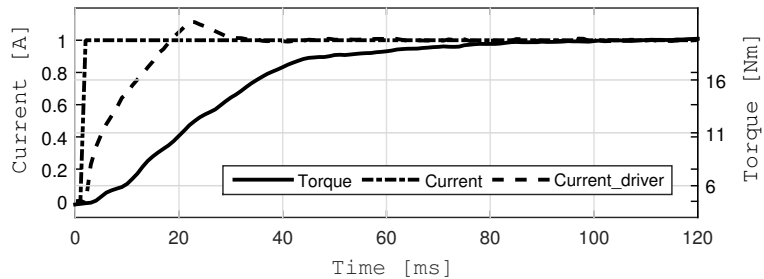

(a)

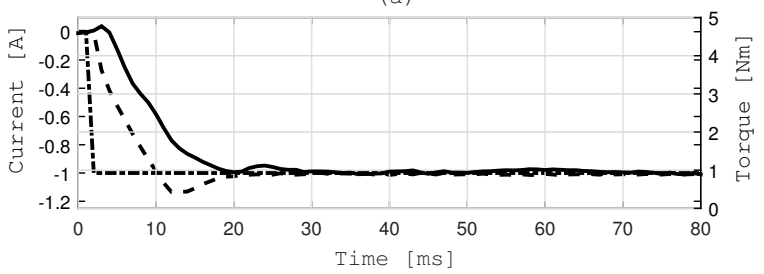

(b)

Fig. 10. Transient performance of the HMR clutch for input current of (a) 0 A to $1 \mathrm{~A}$, (b) $0 \mathrm{~A}$ to $-1 \mathrm{~A}$.

the transmitted torque of the prototype HMR clutch versus the applied current and compares the results with theoretical values obtained using (6) and the FE model. The results agree in magnitude for both minimum and maximum torque values. The minimum transmitted torque is nearly $1.3 \mathrm{Nm}$ which is due to the the friction of the sealing. It is clear that the FE model is not able to capture the hysteresis effect of the HMR clutch. Like other ferromagnetic devices, HMR clutches show nonlinear hysteresis relationship between the input current and the output torque of the clutch [23]. This hysteresis is a rate dependent characteristic that can change with frequency [24]. The use of magnetic field measurement using embedded Hall sensors within the clutch pack for compensating hysteresis was proposed first in [25]. The effectiveness of this method for modeling and implementing high fidelity torque control in both conventional and HMR clutches was studied in [24], [26][29]. The shortcoming of the FE model in capturing hysteresis effects will not be detrimental to the results and is ignored in the current study.

To evaluate the transient performance of the prototype HMR clutch, the output torque was obtained in response to a step input current. The experimental results for both positive (i.e., 1 A) and negative (i.e., -1 A) step inputs are shown in Fig. 10. The solid line represents the measured output torque, the dashdot line is the command input current, and the dashed line 


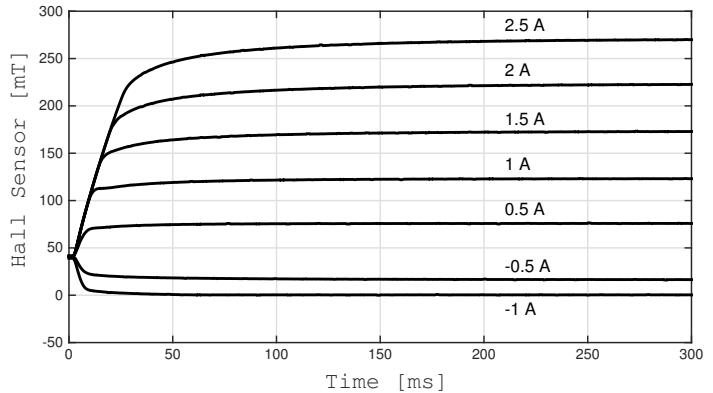

Fig. 11. Magnetic flux density to step inputs in the HMR clutch.

is the applied current of the driving electronics. The output torque at $0 \mathrm{~A}$ current is near $4 \mathrm{Nm}$ due to the use of the permanent magnet. The output torque has a relatively short rise time of about $40 \mathrm{~ms}$, and $15 \mathrm{~ms}$ for the step input current of $0 \mathrm{~A}$ to $1 \mathrm{~A}$, and $0 \mathrm{~A}$ to $-1 \mathrm{~A}$, respectively. As discussed in [30], the response time of MR devices in general depends on the input rotational velocity, current, and inductance of the electromagnetic coil. In order to gain more insights on the effect of the input current on the magnetic field propagation and the magnetic rise time of the HMR clutch, seven step input currents with magnitudes ranging from $-1 \mathrm{~A}$ to $2.5 \mathrm{~A}$ were applied to the clutch and the corresponding magnetic field responses were measured experimentally using the embedded Hall sensors. The results are shown in Fig. 11. These results clearly demonstrate the dependency of the magnetic field propagation to the applied input current. As observed, the rise time increases as the magnitude of the step input current increases. This phenomenon can be partially explained by the identical initial slopes observed in all curves. This initial slope is determined by the voltage constraint imposed by the electronics used to provide the command currents.

To further validate this observation, the transient behavior of the prototype HMR clutch was compared to two conventional MR clutches which were previously developed in our research group. The normalized magnetic flux density of each clutch to a 1 A step input current is depicted in Fig. 12. As seen, the responses of all three clutches have the same initial slope. This validates our earlier observation regarding inevitable restrictions imposed by the electronic drivers. The results also show the benefit of using permanent magnets in the HMR clutch. It is clear that despite an identical starting slope the HMR clutch has the best time constant among three prototypes.

The closed-loop performance of the prototype HMR clutch and its dynamic characteristics was also evaluated using a PID controller. A dSAPCE DS 1103 was used to provide (command) current to the prototype HMR clutch in response to the closed-loop feedback error between the desired and actual output torques. Fig. 13 shows the closed-loop block diagram. The PID controller gains were obtained experimentally to achieve a satisfactory bandwidth and accurate torque tracking.

We used this configuration to obtain the closed-loop frequency response of the HMR clutch. A sinusoidal reference torque sweeping from $0.5 \mathrm{~Hz}$ to $50 \mathrm{~Hz}$ was used to measure the output torque of the HMR clutch with the load cell.

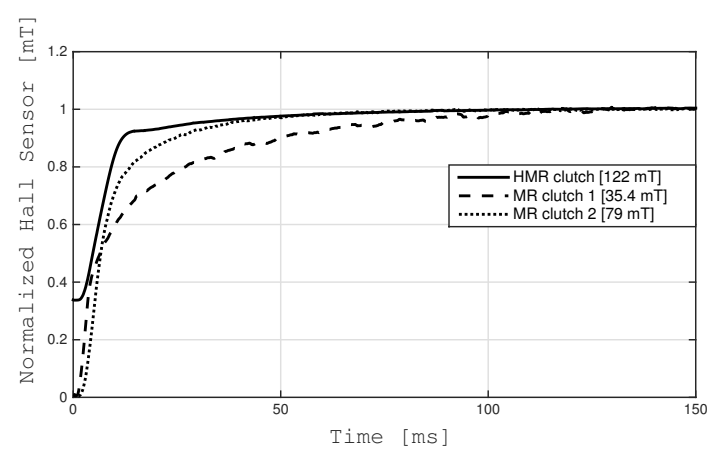

Fig. 12. Normalized magnetic flux density of the prototype HMR clutch and two conventional coil-based MR clutches to a 1 A step input current.

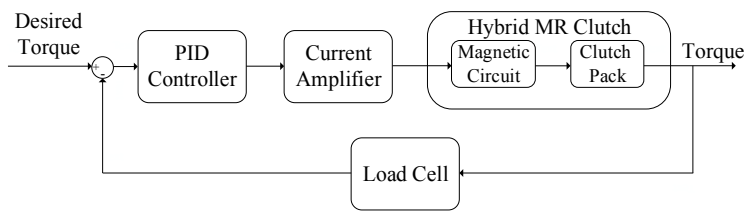

Fig. 13. Closed-loop control block diagram.

Fig. 14 illustrates the frequency response of the prototype HMR clutch. As observed, using the 3-dB power loss cutoff frequency definition, the system bandwidth is measured to be approximately $30 \mathrm{~Hz}$. The proposed actuator, despite its non-optimized controller, offers much higher bandwidth than conventional geared or direct-drive actuators.

The performance of the HMR clutch was further evaluated in time domain using a rapidly varying multi-sinusoidal signal as the reference torque value. The reference signal included three sinusoidal signals with frequencies of 1,5 , and $10 \mathrm{~Hz}$, equal phase shift of 270 degrees, and equal magnitude of 1 $\mathrm{Nm}$, as well as a DC component of $15 \mathrm{Nm}$. Fig. 15 depicts the desired torque signal and compares it with the output torque of the clutch. As seen, the HMR clutch is capable of achieving fast and accurate torque tracking control. The results clearly validate the large control bandwidth of the HMR clutch as a fast and responsive actuation mechanism.
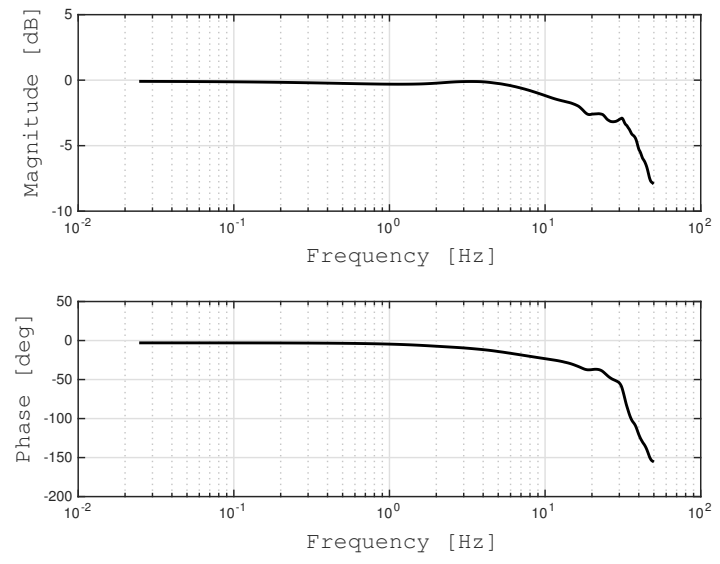

Fig. 14. Closed loop frequency response of the prototype HMR clutch. 


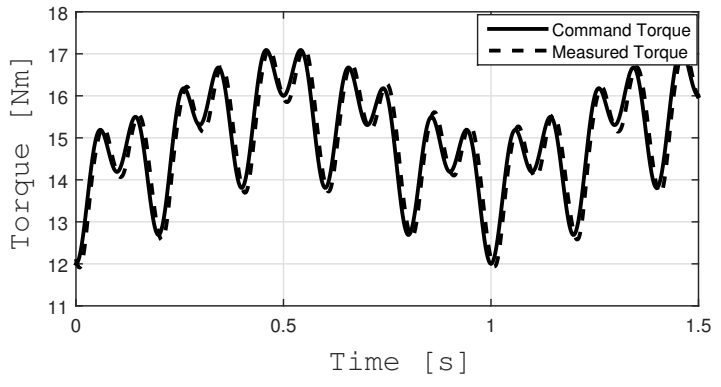

Fig. 15. Torque tracking of a multi-sinusoidal reference.

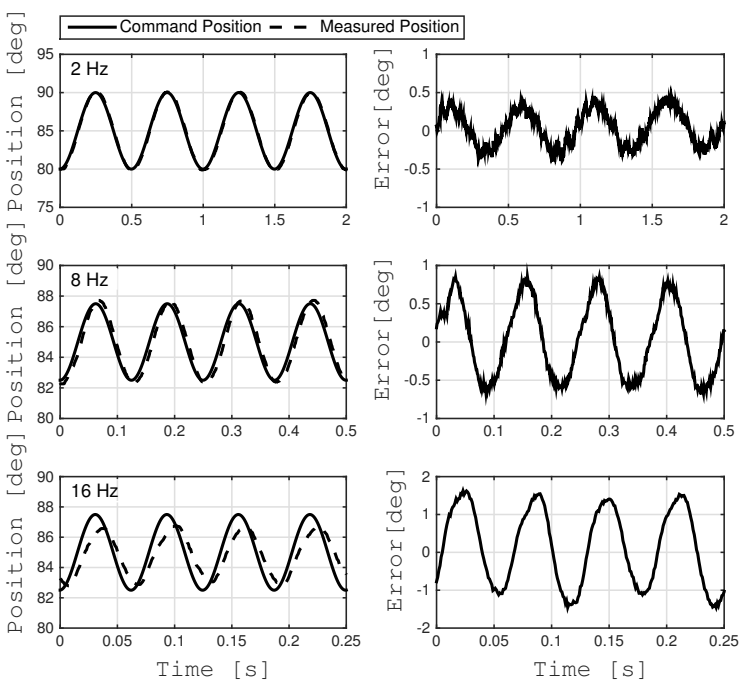

Fig. 16. Position tracking using a pair of antagonistically coupled HMR and MR clutch.

The prototype HMR clutch was paired with a previously developed conventional MR clutch [11] to evaluate the performance of the HMR clutch in an antagonistic configuration. In this experiment, the motor provided rotational velocity in opposite directions using appropriate gearing enabling the pair of HMR and MR clutches to drive the output shaft antagonistically in either directions (see Fig. 8). One should note that the pair of clutches used in this antagonistic configuration had very different characteristics. Nonetheless, high-performance position control was achieved. A trajectory tracking experiment was conducted using an angular encoder mounted on the shaft to provide the feedback signal. Fig. 16 shows the trajectory tracking response obtained using this actuation paradigm to a referenced position command. Similar results in the presence of external disturbances are shown in Fig. 17. As seen, this actuation mechanism provides excellent disturbance rejection and high degrees of compliance. The simplicity of control and back-drivability of the actuator are important properties essential for human-robot collaborations.

\section{CONCLUSION}

In this paper, we presented a lightweight MagnetoRheological clutch with hybrid magnetization, called Hybrid

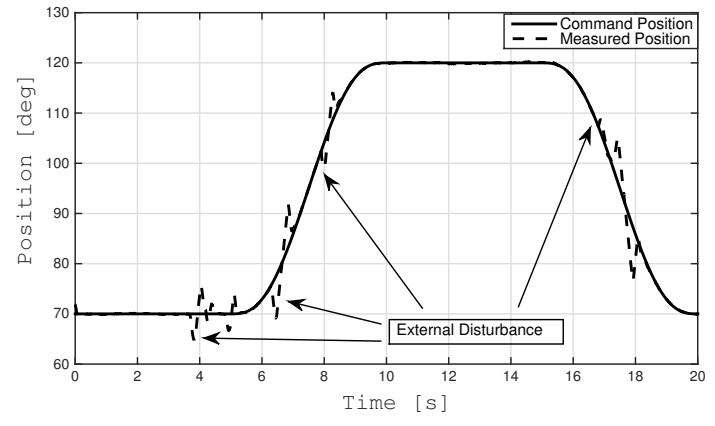

Fig. 17. Position control in the presence of external disturbances.

Magneto-Rheological (HMR) clutch. The hybrid magnetization utilized a combination of a permanent magnet and an electromagnetic coil for providing magnetic field and reducing magnetic saturation in the magnetic circuit. By avoiding saturation we were able to significantly reduce the volume of the material used in the magnetic path, hence the total mass of the clutch for a given nominal torque.

This study exploited the use of a multi-objective optimization to facilitate the design of the HMR clutch. An optimal solution was achieved that allowed to generate both maximum magnetic flux density and absolute zero magnetic flux density within the clutch pack using hybrid magnetization, while minimizing the total mass of the HMR clutch.

Extensive experimental results validated the expected transient and dynamic behavior of the prototype HMR clutch. The results clearly demonstrated the benefits of the hybrid magnetization in improving the transient behavior and reducing the overall mass of these devices. The lightweight design of the HMR clutch and inherent back-drivability of these devices make them an ideal candidate for robotic applications requiring fast and precise motion and torque control.

\section{REFERENCES}

[1] M. T. Avraam, "MR-fluid brake design and its application to a portable muscular rehabilitation device," Ph.D. dissertation, Univ. Libre Bruxelles, Brussels, Belgium, 2009.

[2] W. Li, P. Yadmellat, and M. R. Kermani, "Design optimization and comparison of magneto-rheological actuators," in 2014 IEEE International Conference on Robotics and Automation (ICRA). IEEE, 2014, pp. 5050-5055.

[3] C. Rossa, A. Jaegy, J. Lozada, and A. Micaelli, "Design considerations for magnetorheological brakes," IEEE/ASME Transactions on Mechatronics, vol. 19, no. 5, pp. 1669-1680, 2013.

[4] K. Karakoc, E. J. Park, and A. Suleman, "Design considerations for an automotive magnetorheological brake," Mechatronics, vol. 18, no. 8, pp. 434-447, 2008.

[5] Q.-H. Nguyen and S.-B. Choi, "Optimal design of a vehicle magnetorheological damper considering the damping force and dynamic range," Smart materials and Structures, vol. 18, no. 1, p. 015013, 2008.

[6] Q.-H. Nguyen, S.-B. Choi, and N. M. Wereley, "Optimal design of magnetorheological valves via a finite element method considering control energy and a time constant," Smart Materials and Structures, vol. 17, no. 2, p. 025024, 2008.

[7] D. Wang, Z. Tian, Q. Meng, and Y. Hou, "Development of a novel twolayer multiplate magnetorheological clutch for high-power applications," Smart Materials and Structures, vol. 22, no. 8, p. 085018, 2013.

[8] F. Gratzer, H. Steinwender, and A. Kušej, "Magnetorheological AWD clutches," ATZautotechnology, vol. 8, no. 10, pp. 32-37, 2008. 
[9] E. J. Park, D. Stoikov, L. F. da Luz, and A. Suleman, "A performance evaluation of an automotive magnetorheological brake design with a sliding mode controller," Mechatronics, vol. 16, no. 7, pp. 405-416, 2006.

[10] T. Kikuchi, K. Ikeda, K. Otsuki, T. Kakehashi, and J. Furusho, "Compact MR fluid clutch device for human-friendly actuator," in Journal of Physics: Conference Series, vol. 149, no. 1. IOP Publishing, 2009, p. 012059.

[11] A. S. Shafer and M. R. Kermani, "On the feasibility and suitability of MR fluid clutches in human-friendly manipulators," IEEE/ASME Transactions on Mechatronics, vol. 16, no. 6, pp. 1073-1082, 2010.

[12] T. Kikuchi, K. Otsuki, J. Furusho, H. Abe, J. Noma, M. Naito, and N. Lauzier, "Development of a compact magnetorheological fluid clutch for human-friendly actuator," Advanced Robotics, vol. 24, no. 10, pp. $1489-1502,2010$.

[13] P. Yadmellat, A. S. Shafer, and M. R. Kermani, "Design and development of a single-motor, two-DOF, safe manipulator," IEEE/ASME Transactions on Mechatronics, vol. 19, no. 4, pp. 1384-1391, 2013.

[14] P. Fauteux, M. Lauria, B. Heintz, and F. Michaud, "Dual-differential rheological actuator for high-performance physical robotic interaction," IEEE Transactions on Robotics, vol. 26, no. 4, pp. 607-618, 2010.

[15] F. Bucchi, P. Forte, F. Frendo, A. Musolino, and R. Rizzo, "A failsafe magnetorheological clutch excited by permanent magnets for the disengagement of automotive auxiliaries," Journal of Intelligent Material Systems and Structures, vol. 25, no. 16, pp. 2102-2114, 2014.

[16] G. Aydar, X. Wang, and F. Gordaninejad, "A novel two-way-controllable magneto-rheological fluid damper," Smart Materials and Structures, vol. 19 , no. 6 , p. 065024,2010

[17] T. Saito and H. Ikeda, "Development of normally closed type of magnetorheological clutch and its application to safe torque control system of human-collaborative robot," Journal of Intelligent Material Systems and Structures, vol. 18, no. 12, pp. 1181-1185, 2007.

[18] H. Böse, T. Gerlach, and J. Ehrlich, "Magnetorheological torque transmission devices with permanent magnets," in Journal of Physics: Conference Series, vol. 412, no. 1. IOP Publishing, 2013, p. 012050.

[19] F. Bucchi, P. Forte, and F. Frendo, "Geometry optimization of a magnetorheological clutch operated by coils," Proceedings of the Institution of Mechanical Engineers, Part L: Journal of Materials: Design and Applications, vol. 231, no. 1-2, pp. 100-112, 2017.

[20] M. Moghani and M. R. Kermani, "Design and development of a hybrid magneto-rheological clutch for safe robotic applications," in 2016 IEEE International Conference on Robotics and Automation (ICRA). IEEE, 2016, pp. 3083-3088.

[21] R. W. Phillips, "Engineering applications of fluids with a variable yield stress," Ph.D. dissertation, Univ. California, Berkeley, CA, 1969.

[22] J. D. Carlson, "MR fluids and devices in the real world," International Journal of Modern Physics B, vol. 19, no. 07n09, pp. 1463-1470, 2005.

[23] P. Yadmellat, A. S. Shafer, and M. R. Kermani, "Design and development of a safe robot manipulator using a new actuation concept," in Robotics and Automation (ICRA), 2013 IEEE International Conference on. IEEE, 2013, pp. 337-342.

[24] M. Moghani and M. R. Kermani, "Hysteresis modeling of a hybrid magneto-rheological actuator," in 2016 IEEE International Conference on Advanced Intelligent Mechatronics (AIM). IEEE, 2016, pp. 246-251.

[25] A. Shafer and M. R. Kermani, "Magneto-rheological clutch with sensors measuring electromagnetic field strength," Jan. 10 2017, US Patent $9,539,731$

[26] P. Yadmellat and M. R. Kermani, "Adaptive control of a hysteretic magnetorheological robot actuator," IEEE/ASME Transactions on Mechatronics, vol. 21, no. 3, pp. 1336-1344, 2016.

[27] — "Study of limit cycle in antagonistically coupled magnetorheological actuators," Control Engineering Practice, vol. 35, pp. 92 101,2015

[28] _ "Adaptive hysteresis compensation for a magneto-rheological robot actuator," in 2013 IEEE/RSJ International Conference on Intelligent Robots and Systems. IEEE, 2013, pp. 4900-4905.

[29] W. Li, P. Yadmellat, and M. R. Kermani, "Linearized torque actuation using FPGA-controlled magnetorheological actuators," IEEE/ASME Transactions on Mechatronics, vol. 20, no. 2, pp. 696-704, 2014

[30] N. C. Kavlicoglu, B. M. Kavlicoglu, Y. Liu, C. A. Evrensel, A. Fuchs, G. Korol, and F. Gordaninejad, "Response time and performance of a high-torque magneto-rheological fluid limited slip differential clutch," Smart materials and structures, vol. 16, no. 1, p. 149, 2007.

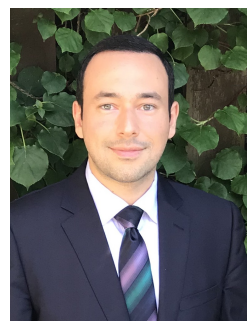

Masoud Moghani received the M.E.Sc. degree in electrical and computer engineering from the University of Western Ontario, London, ON, Canada in 2016.

His research interests include soft robotics, and artificial intelligence.

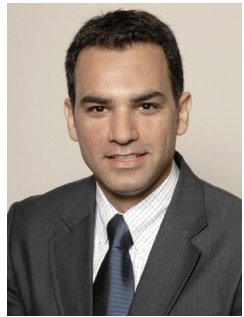

Mehrdad R. Kermani (IEEE member, 05) received his $\mathrm{Ph} . \mathrm{D}$. degree from the University of Western Ontario (UWO), London, ON, Canada in 2005 in Robotics, followed by a Post-Doctoral Fellowship award from Natural Sciences and Engineering Research Council of Canada (NSERC). In 2006, he joined the Department of Electrical and Computer Engineering, UWO, where he is currently an Associate Professor. His research interests include collaborative robotics, human-centric technologies for industrial robots, smart actuators, and sensors

Dr. Kermani is an active member of Earth \& Space Institute, a member of Robotic and Automation Society (RA), and a Professional Engineer (P.Eng.) in the Province of Ontario. 